\title{
Modelling the effect of variable density and diffusion coefficient on heat and mass transfer from a single component spherical drop evaporating
}

\author{
in high temperature air streams
}

\author{
S. Tonini, G.E. Cossali
}

September 27, 2017

\begin{abstract}
Species, energy and momentum conservation equations are solved in spherical symmetry and under ideal gas approximation, to yield an analytical model capable to evaluate the heat transfer and the evaporation rate from a single component spherical drop under quasi-steady conditions, accounting for the temperature dependence of mixture density and diffusion coefficient. The model is applied to predict the evaporation rate from water, acetone, ethanol and three hydrocarbon droplets under temperature conditions of interest for applicative fields, like fire control and combustion. The results from the proposed model are compared with those obtained by the classical model where mass diffusion coefficient and gas density are kept constant to an average value. The proposed model is validated against experimental data from the open literature of transient drop evaporation in high temperature air streams.
\end{abstract}




\section{Nomenclature}

c Molar density

$c_{p g} \quad$ Gas mixture specific heat at constant pressure

$c_{p v} \quad$ Vapour specific heat at constant pressure

$D_{d} \quad$ Drop diameter

$D_{10} \quad$ Mass diffusion coefficient

F $\quad$ Function, equation (33)

$H \quad$ Logarithm of gas molar fraction

$k \quad$ Thermal conductivity

$K_{0} \quad$ Non-dimensional coefficient, equation (5)

Le Lewis number

$L e^{M} \quad$ Modified Lewis number

$m_{e v} \quad$ Evaporation rate

Mm Molar mass

$N u \quad$ Nusselt number

Pr Prandtl number

$P_{T} \quad$ Pressure

$q_{d} \quad$ Drop heating-up heat rate

$r \quad$ Radial coordinate

$\bar{R} \quad$ Universal gas constant

$R_{0} \quad$ Drop radius

Sc Schmidt number

$S c^{M} \quad$ Modified Schmidt number

T Temperature

$U \quad V$ elocity

y Molar fraction

$Y \quad$ Non-dimensional evaporation rate
$K$

$\mathrm{kmol} / \mathrm{m}^{3}$

$J / k g K$

$J / k g K$

m

$m^{2} / s$

$W / m K$

$\mathrm{kg} / \mathrm{s}$

$\mathrm{kg} / \mathrm{kmol}$

$P a$

$W$

m

$k g / k m o l K$

m

$\mathrm{m} / \mathrm{s}$ 
Greek symbols

$\alpha_{\text {ref }} \quad$ Averaging parameter

$\chi \quad M$ ass fraction

$\rho \quad$ Density

$k g / m^{3}$

$\Lambda \quad$ Non-dimensional number (equation 16)

$\theta \quad$ Molar mass ratio $\theta=\frac{M m^{(1)}-M m^{(0)}}{M m^{(0)}}$

$\zeta \quad$ Non-dimensional radial coordinate: $\zeta=R_{0} / r$

$\Psi \quad$ Evaporation rate ratio, $\Psi_{*}=\frac{Y_{*}}{Y_{7 / 4}}$

Subscripts

$r \quad$ Radial component

ref Reference condition

$s \quad$ Drop surface

$\infty \quad$ Free stream conditions

$0 \quad$ Ambient or reference

Superscripts

c Constant mass density

(0) Gas

(1) Vapour

$\sim$ Non-dimensional

\section{Introduction}

Evaporation processes are quite common in a wide range of engineering and industrial applications, and many of them, like spray drying, spray cooling, spray combustion, fire suppression, etc., rely on simultaneous heat and mass transfer from a drop in a gaseous environment. Modelling of these processes has been the subject of a large number of investigations, also due to the many complex physical phenomena involved, and a complete review can be find in [1], [2]. Modern analysis of spray heating and evaporation is performed by numerical simulation through CFD methodologies and detailed models of single drop heating and evaporation have to be simplified to become CPU 
efficient in multi-particle analysis. This need urged the introduction of many simplifying hypotheses to develop analytical models that are nowadays implemented in many commercial and in-house codes. Approximations like quasi-steadiness, drop sphericity, constant properties of the gas mixture, to cite the most important, are usually assumed (see for example [2]), although recently, attempts to relieve some of them became available in the open literature [3], [4], [5], [6].

Mass and heat transfer occurring within the liquid phase of an evaporating drop in hot environment has been widely investigated. The simplest models, that are implemented in many conventional CFD codes for spray applications, neglect temperature gradient inside the drop, thus assuming an infinite liquid thermal conductivity [7], on the basis of the existence of liquid recirculation inside the drop (Hill-vortex) that strongly enhances temperature homogeneisation. However experimental evidences [8], [9] questioned the applicability of this assumption and to account for this effect a finite effective thermal conductivity is often assumed, which takes into account both conduction and convective transport due to liquid recirculation inside the drop [7], [1].

Drop radius shrinking due to evaporation sets a moving boundary problem when energy and species conservation equations have to be solved, but the totality of the analytical evaporation models implemented in commercial CFD codes neglects this effect. Only recently this effect has been considered by Sazhin et al. in [5], [6], who included the effect of a moving boundary to solve the conservation equations in the liquid phase, and a still manageable equation was proposed that could be implemented in a CFD code [10]. Accounting for drop shrinking has a non neglectful effect on liquid temperature prediction and yields longer evaporation times when compared to the conventional approach.

When a drop is injected into a hot gas, large temperature gradients in the gas phase are achieved and the commonly accepted constant property approximation becomes questionable since thermophysical properties, among which density and diffusion coefficients, have a strong dependence on temperature. This problem is usually circumvented through the use of an average value for all thermophysical properties calculated at a reference temperature, commonly obtained by the ' $1 / 3$-rd rule' [11], but this may not be enough to correctly catch the effect. As already stressed in [2] back in 1989, the prediction of "the drop vaporisation rate is extremely sensitive to the method used for the evaluation of physical properties" and this motivates the necessity to properly calculate them. A model that takes into account gas density dependence on temperature, and then accounting for density gradient effects, may yield significant differences respect to the classical ones under high gas temperature conditions, and it may 
better perform when compared with available experimental results [3]. But gas density is not the only property that significantly varies with temperature, the vapour diffusion coefficient in a gaseous mixture also shows a significant temperature dependence. Statistical thermodynamics arguments [12] and existing empirical correlations [13] suggest a power law dependence on the absolute temperature with exponent ranging from $3 / 2$ to $7 / 4$.

The temperature dependence of all the involved thermophysical properties is usually accounted for in numerical studies of drop heating and evaporation, and these models have been deeply refined over the decades including many physical aspects like the effect of deformation, drop composition, interaction with other drops and/or solid surfaces (see [14], [15], [16] and[17] for recent references on droplet evaporation using the VOF methodology to capture the $3 \mathrm{D}$ droplet evolution). It is self-evident that the complexity of the numerical implementation and the CPU time required for a single drop test case simulation, does not allow the use of these model in multi-particle simulations, although they can be used as reliable benchmarking for simpler models to be developed.

The main aim of this work is to develop an evaporation model for single component drops that includes the effect of gas density and diffusion coefficient temperature dependence, with the target of efficiently implementing it in comprehensive CFD tools for predicting the evolution of sprays injected in hot environment. The next section describes the mathematical modelling adopted in the present investigation and the derivation of the proposed analytical solutions of the energy and species conservation equations, accounting for the effect of convection. The results from the parametrical analysis performed under steady-state conditions to compare the present model with the classical one that assumes constant thermophysical properties are then discussed, followed by the validation of the proposed model against various data bases of experimental measurements of transient drop evaporation in almost quiescent and convective environment. Finally the main conclusions are briefly summarised.

\section{Model Equations}

The model described below retains some important approximations that characterise the majority of existing models for heating and evaporation of a liquid drop, like spherical symmetry and quasi-steadiness among others, while relieving two important limitations, namely the constancy of the gas density and of the gas diffusivity coefficients. The steady-state energy, momentum and species conservation equations will then be written in spherical symmetry 
and analytical solutions will be presented. The energy conservation equation, neglecting minor terms like dissipation by viscous stresses, species excess kinetic energy and work pressure forces and assuming constant vapour heat capacity and thermal conductivity can be written as (see [18] for the complete equation):

$$
\rho U c_{p v} \frac{d T}{d r}=k\left(\frac{d^{2} T}{d r^{2}}+\frac{2}{r} \frac{d T}{d r}\right)
$$

where $c_{p v}$ is the vapour specific heat. This equation can be solved independently from species and momentum conservation equations, just accounting for mass conservation that yields a relation between the velocity $U$ and the evaporation rate under the form:

$$
\rho U=\frac{m_{e v}}{4 \pi r^{2}}
$$

where $m_{e v}$ is the evaporation rate. The well known analytic solution of equation (1) with Dirichlet boundary conditions:

$$
T\left(r=R_{0}\right)=T_{s} ; T(r=\infty)=T_{\infty}
$$

yields the temperature field across the gas phase:

$$
\tilde{T}(\zeta)=\left(1-K_{0}\right) e^{-Y \zeta}+K_{0}
$$

where $\zeta=\frac{R_{0}}{r}$ and $\tilde{T}=\frac{T}{T_{\infty}}, Y=\frac{m_{e v} c_{p, r e f}}{4 \pi R_{0} k_{r e f}}$ and

$$
K_{0}=\frac{\tilde{T}_{s}-e^{-Y}}{1-e^{-Y}}
$$

In this solution $c_{p, r e f}$ and $k_{r e f}$ are considered to be constant and evaluated at some reference conditions, which are usually defined as:

$$
T_{r e f}=\alpha_{r e f} T_{\infty}+\left(1-\alpha_{r e f}\right) T_{s} ; \quad \chi_{r e f}^{(1)}=\alpha_{r e f} \chi_{\infty}^{(1)}+\left(1-\alpha_{r e f}\right) \chi_{s}^{(1)}
$$

where $\chi^{(1)}$ is the mass fraction of the evaporating species. The classical value for $\alpha_{r e f}$ is $1 / 2$, but often the value $\alpha_{r e f}=1 / 3$, suggested in [11] (the so-called "1/3 rd rule") is used, as in [2] for example.

The molar species fluxes can be written as:

$$
N_{j}^{(p)}=N_{j}^{(T)} y^{(p)}-c D_{10} \nabla_{j} y^{(p)}
$$

where $p=0$ stands for the gas species and $p=1$ for the evaporating species, $N_{j}^{(T)}=N_{j}^{(1)}+N_{j}^{(0)}, y^{(p)}$ is the molar fraction of the species $p$ and $c$ is the molar density. To notice that the usual way to approach this problem is by 
using a mass form of the species conservation equations (see [2], [19]), but it can be shown (see [20]) that for single component drops the two approaches are equivalent.

Under the assumption of stationary liquid-gas interface and pure liquid (i.e. absence of diffusion of the component 0 into the liquid drop) the species conservation equations for a single component spherical drop steadily evaporating in a hot gaseous environment are:

$$
\begin{aligned}
& N_{r}^{(T)} y^{(0)}-c D_{10} \frac{d y^{(0)}}{d r}=0 \\
& N_{r}^{(T)} y^{(1)}-c D_{10} \frac{d y^{(1)}}{d r}=\frac{m_{e v}}{M m^{(1)} 4 \pi r^{2}}
\end{aligned}
$$

where $D_{10}$ is the binary diffusion coefficient and $M m^{(p)}$ is the molar mass. To notice that the second equation can be found from the first one and the mass conservation equation (2), since $y^{(0)}+y^{(1)}=1$.

The momentum conservation equation for the evaporating drop, under the same simplifying hypotheses and assuming constant viscosity, as in [21], is:

$$
\rho U \frac{\partial U}{\partial r}=-\frac{\partial P_{T}}{\partial r}+\mu\left(\frac{\partial^{2} U}{\partial r^{2}}+\frac{2}{r} \frac{\partial U}{\partial r}\right)
$$

The novelty of the present model relies on the fact that both gas density and binary mass diffusion coefficient will be assumed to vary. The temperature and pressure dependence of molar gas density is that found for an ideal gas:

$$
\tilde{c}=\frac{c}{c_{r e f}}=\frac{P_{T}}{c_{r e f} R T_{\infty} \tilde{T}}=\frac{\check{P}_{T}}{\tilde{T}}
$$

where: $\check{P}_{T}=\frac{P_{T}}{c_{r e f} R T_{\infty}}$, while $D_{10}$ is expected to depend on temperature following the general rule:

$$
\tilde{D}=\frac{D_{10}}{D_{10, \text { ref }}}=\frac{\tilde{T}^{n}}{\tilde{T}_{r e f}^{n}}
$$

where $D_{10, \text { ref }}=D_{10}\left(T_{\text {ref }}\right)$ is a reference value. In equation (12), $n=0$ stands for the case of constant diffusion coefficient, which is the usual assumption that yields the classical results [2], [19], $n=3 / 2$ is the value suggested by classical statistical thermodynamics [12] and $n=7 / 4$, is the values given by the widely used FSG correlation [13]. To notice that the mass density depends also on composition since for an ideal binary mixture:

$$
\rho=\frac{P_{T} M m^{(0)}}{R T}\left(1+\theta y^{(1)}\right)
$$

with $\theta=\frac{M m^{(1)}-M m^{(0)}}{M m^{(0)}}$, and this is the reason why the molar approach is preferred, yielding a simpler form of the final solutions, but again it must be stressed that the two approaches (molar and mass forms of the species conservation equations) are equivalent [20]. 


\subsection{Analytic solutions of the model equations}

Introducing the following non-dimensional variables:

$$
H=\ln \left(y^{(0)}\right) ; \check{U}=U \frac{R_{0}}{D_{10, \text { ref }}}
$$

and the nondimensional pressure $\left(\check{P}_{T}=\frac{P_{T}}{c_{r e f} R T_{\infty}}\right)$, the modified Schmidt $\left(S c^{M}=\frac{\mu}{D_{10, r e f} c_{r e f} M m_{v}}\right)$ and Lewis $\left(L e^{M}=\frac{k_{r e f}}{c_{p, r e f} D_{10, r e f} M_{m v} c_{r e f}}\right)$ numbers and the parameter: $\Lambda=\frac{R T_{\infty} R_{0}^{2}}{M_{m v} D_{10, r e f}^{2}}$ the above reported species and momentum conservation equations reduce to:

$$
\begin{aligned}
\tilde{c} \tilde{D} \frac{d H}{d \zeta} & =-L e^{M} Y \\
-\frac{\partial \check{P}_{T}}{\partial \zeta} & =\frac{\zeta^{2}}{\Lambda}\left(Y L e^{M} \frac{\partial \check{U}}{\partial \zeta}+S c^{M} \frac{d^{2} \check{U}}{d \zeta^{2}}\right)
\end{aligned}
$$

It has already been shown [21] that for a large variety of conditions of interest for applications, the non-dimensional parameter $\Lambda$ assumes quite large values, then justifying the use of an asymptotic form (for $\Lambda \rightarrow \infty$ ) of the momentum equation, that in the present case yields: $\frac{d \check{P}_{T}}{d \zeta}=0$.

Since in this case: $P_{T}=P_{T, \infty}$, under the assumption of ideal gas behaviour for the gaseous mixture, equation (11) can be written as:

$$
\tilde{c} \tilde{T}=\check{P}_{T, \infty}=\frac{c_{\infty}}{c_{r e f}}=\frac{T_{r e f}}{T_{\infty}}=\tilde{T}_{r e f}
$$

and equation (15) can be transformed, using equations (11) and (12) into the following ODE:

$$
\frac{d H}{d \zeta}=-\left(Y L e^{M} \tilde{T}_{r e f}^{n-1}\right) \tilde{T}^{1-n}
$$

where the function $\tilde{T}(\zeta)$ is given by equation (4).The analytic solution of equation (18) exists for many rational values of the parameter $n$ (those for which the integral $I_{n}(\zeta)=\int \tilde{T}^{1-n} d \zeta$ can be analytically evaluated). Applying the Dirichlet boundary conditions:

$$
H(0)=\ln y_{\infty}^{(0)}=\ln \left(1-y_{\infty}^{(1)}\right) ; \quad H(1)=\ln y_{s}^{(0)}=\ln \left(1-y_{s}^{(1)}\right)
$$

the following equation can be derived:

$$
\frac{1}{L e^{M} \tilde{T}_{r e f}^{n-1}} \ln \left(1+B_{M}^{*}\right)=Y \int_{0}^{1} \tilde{T}^{1-n} d \zeta=F_{n}\left(K_{0}, \tilde{T}_{s}\right)
$$


where $B_{M}^{*}=\frac{y_{s}^{(1)}-y_{\infty}^{(1)}}{1-y_{s}^{(1)}}$ and the definite integral $F_{n}\left(K_{0}, \tilde{T}_{s}\right)=Y \int_{0}^{1} \tilde{T}^{1-n} d \zeta$ can conveniently be written in terms of the parameters $K_{0}$ instead of $Y$, since its functional form depends on the sign of $K_{0}$ for $n \neq 0$ (see Appendix).

Once the analytic form of $F_{n}\left(K_{0}, \tilde{T}_{s}\right)$ is substituted into equation (20), the solution of the non-linear equation allows to evaluate $K_{0}=K_{0}\left(y_{v, s}, y_{v, \infty}, \tilde{T}_{s}, L e^{M}\right)$ and the evaporation rate can be calculated from the definition of $K_{0}$ (equation 5):

$$
m_{e v}=4 \pi R_{0} \frac{k_{r e f}}{c_{p, r e f}} \ln \frac{K_{0}-1}{K_{0}-\tilde{T}_{s}}
$$

From equation (4) the heat rate, defined positive when entering the drop, can then be evaluated:

$$
Q=4 \pi R_{0}^{2} k_{r e f}\left(\frac{\partial T}{\partial r}\right)_{r=R_{0}}=4 \pi R_{0} k_{r e f}\left(T_{\infty}-T_{s}\right) \frac{Y}{e^{Y}-1}
$$

It should be noticed that the present approach yields an implicit equation in the evaporation rate (20), contrary to the classical approach [2], where the evaporation rate is calculated in an explicit form; on the other hand the classical model [2] provides the heat rate in an implicit form, while in the present model this is given explicitly by equation (22). Then, the computation effort of the present model is comparable to that of the classical model, with the advantage that the temperature dependence of density and diffusion coefficient is fully accounted for.

\section{Effect of convection}

The effect of convection is taken into account following the film theory approach (see [22]), which is the method used in [2] to derive that model. Two different partially overlapping regions are defined for the solution of the heat and mass transfer problems, defined by a thermal $\left(R_{2}-R_{0}\right)$ and a diffusional $\left(R_{3}-R_{0}\right)$ thickness and the boundary conditions of the free stream are imposed on these boundaries. The heat equation is then solved with boundary conditions:

$$
T(\zeta=1)=T_{s} ; T\left(\zeta=\zeta_{2}\right)=T_{\infty}
$$

where $\zeta_{2}=\frac{R_{2}}{R_{0}}$, yielding:

$$
\widetilde{T}=\left(1-\tilde{T}_{s}\right) \frac{e^{-Y \zeta}-e^{-Y}}{e^{-Y \zeta_{2}}-e^{-Y}}+\tilde{T}_{s}
$$

With the change of variables: $\check{Y}=Y\left(1-\zeta_{2}\right)$ and $\eta=\frac{\zeta-\zeta_{2}}{1-\zeta_{2}}$, equation (24) can be re-written as:

$$
\widetilde{T}=\left(1-K_{\zeta_{2}}\right) e^{-\check{Y} \eta}+K_{\zeta_{2}}
$$


where $K_{\zeta_{2}}=\frac{\tilde{T}_{s}-e^{-\check{Y}}}{1-e^{-\check{Y}}}$,and equation (18) becomes:

$$
\frac{d H}{d \eta}=-\left(\check{Y} L e^{M} \tilde{T}_{r e f}^{n-1}\right) \tilde{T}^{1-n}
$$

Imposing the B.C.:

$$
H\left(\eta_{3}\right)=\ln y_{\infty}^{(0)}=\ln \left(1-y_{\infty}^{(1)}\right) ; \quad H(1)=\ln y_{s}^{(0)}=\ln \left(1-y_{s}^{(1)}\right)
$$

where $\eta_{3}=\frac{\zeta_{3}-\zeta_{2}}{1-\zeta_{2}}$, the solution is found as:

$$
\frac{1}{L e^{M} \tilde{T}_{r e f}^{n-1}} \ln \left(1+B_{M}^{*}\right)=\check{Y} \int_{\eta_{3}}^{1} \tilde{T}^{1-n} d \eta
$$

where the integral $\int_{\eta_{3}}^{1} \tilde{T}^{1-n} d \eta$ can be calculated for the already mentioned values of $n$ (see appendix) and equation (28) can be numerically solved to yield the value of the non-dimensional evaporation rate $\check{Y}$. To notice that when $\zeta_{3}<\zeta_{2}$ the lower limit of the integral should be set equal to zero.

The heat rate can again be explicitly obtained, from equation (25), under the form:

$$
Q=4 \pi R_{0}^{2} k_{r e f}\left(T_{\infty}-T_{s}\right) \frac{Y}{e^{Y\left(1-\zeta_{2}\right)}-1}
$$

Equations (28) and (29) contain the nondimensional parameters $\zeta_{2}$ and $\zeta_{3}$ that set the film thicknesses, and a way to define their values (as in [2]) is through a relation with available empirical correlations.

The parameter $\zeta_{3}$, which defines the diffusional film thickness, can be related to the Sherwood number in analogy with the model of Abramzon and Sirignano [2], and as already derived in [3]:

$$
\zeta_{3}=1-\frac{2}{S h^{*}}
$$

assuming again as in [3] that:

$$
S h^{*}=2+\frac{\left(S h_{0}-2\right)}{F_{M}\left(B_{M}\right)}
$$

where $S h_{0}$ is the Sherwood number calculated from the well known Frossling correlation [23], as suggested in [2]:

$$
S h_{0}=2+0.552 \operatorname{Re}_{f}^{1 / 2} S c_{f}^{1 / 3}
$$

and $F_{M}$ is a relation suggested in [2]:

$$
F_{M}\left(B_{M}\right)=\left(1+B_{M}\right)^{0.7} \frac{\ln \left(1+B_{M}\right)}{B_{M}}
$$


Introducing the Nusselt number by the classical definition $N u=\frac{h 2 R_{0}}{k_{r e f}}$, where the heat transfer coefficient $h$ is defined as the ratio between the normal heat flux at drop surface and the temperature difference $\left(T_{\infty}-T_{s}\right)$, equation (29) becomes:

$$
Q=2 \pi R_{0}^{2} k_{r e f}\left(T_{\infty}-T_{s}\right) N u
$$

thus yielding the following relation with $\zeta_{2}$ :

$$
\zeta_{2}=1-\frac{1}{Y} \ln \left(1+\frac{2 Y}{N u}\right)
$$

It should be noticed that $N u$ differs from the modified Nusselt number $N u^{*}$ used in [2] (see equation 11 in that paper), but they can be related through the equation:

$$
N u=N u^{*} \frac{\ln \left(1+B_{T}\right)}{B_{T}}
$$

where $B_{T}$ is the Spalding heat transfer number [2].

As for the Sherwood number, an empirical correlation can be used to evaluate $N u$, and then $\zeta_{2}$ from (35). The choice of the correlation was found to be relevant for the capability of the model to predict heating and evaporation, particularly during the drop heating-up period. In a previous model [3], where only the dependence of the gas density on temperature and composition was considered, the correlation proposed in [24]:

$$
N u=\frac{2+0.6 \operatorname{Re}_{f}^{1 / 2} \operatorname{Pr}_{f}^{1 / 3}}{1+B_{f}}
$$

where $B_{f}=\frac{c_{p g}\left(T_{\infty}-T_{s}\right)}{h_{L V}}, h_{L V}$ is the latent heat of vaporisation and $c_{p g}$ the gas mixture heat capacity, was employed with good results when validation against experimental data, obtained under steady conditions, was performed. However, this correlation shows some weakness when the prediction of the vaporisation during the heating-up period is attempted. It is well known that the sensible heat rate exchanged by an evaporating drop is lower than that exchanged by a non-evaporating one under the same temperature conditions (see equation (22) for the $\operatorname{Re}=0$ case), but when the cold drop positioned in a hot environment begins to heat up, the evaporation rate is relatively small, since the drop temperature is low, while $B_{f}$ is relatively high and equation (36) tends to under estimate the Nusselt number and the sensible heat rate, leading to the prediction of longer heating-up period. The modification proposed in $[25]$, where the denominator was substituted by $\left(1+B_{f}\right)^{0.7}$, only mitigates the problem. This problem was already noticed and Dwyer [26] proposed a modified form, substituting $B_{f}$ with the Spalding number $B_{T}=\frac{c_{f}\left(T_{\infty}-T_{s}\right)}{h_{L V}+\frac{q_{d}}{m_{e v}}}$, where 
the presence of the additional term $\frac{q_{d}}{m_{e v}}$ (where $q_{d}$ is the heat rate needed to heat-up the drop, see [2]) allows to increase the value of the Nusselt number for low vaporisation regimes. The difficulty in using this form is related to the fact that $B_{T}$ depends on the sensible heat rate and then on $N u$ again, thus yielding an implicit form of the correlation.

To contain the computational effort, the easiness of an explicit form must be maintained, and then the correlation used in the present model is a further modification of the correlation [26], where the denominator is now changed to $\left(1+B_{M}\right)^{0.7}$. It should be noticed that this value is often quite close to $\left(1+B_{T}\right)^{0.7}$, due to the well known relationship linking the two Spalding numbers $B_{T}$ and $B_{M}$ (see [2]). The explicit form of the heat rate relationship (34) rises a further problem when convection is considered: the correlation for $N u$ should never yield values smaller than the asymptotic analytical value $N u_{\mathrm{Re}=0}(Y)=\frac{2 Y}{e^{Y}-1}$ (see equation 22), which holds when $\operatorname{Re}=0$. Since this is not always the case for the modified (36) correlation, a further modification was included yielding the final form:

$$
N u=\max \left\{\frac{2+0.6 \operatorname{Re}_{f}^{1 / 2} \operatorname{Pr}_{f}^{1 / 3}}{\left(1+B_{M}\right)^{0.7}}, N u\left(B_{M}\right)\right\}
$$

where $N u\left(B_{M}\right)=\frac{2 \frac{1}{L e} \ln \left(1+B_{M}\right)}{\left(1+B_{M}\right)^{1 / L e}-1}$ is taken as a good explicit approximation of $N u_{\mathrm{Re}=0}(Y)$. Alternative correlations may be suggested, as proposed in [2].

Finally, to calculate $c_{p, r e f}$ and $k_{r e f}$, the reference temperature and composition were evaluated by equations (6) choosing $\alpha_{r e f}=1 / 2$, following [25]; to notice that $\alpha_{r e f}=1 / 3$, suggested by [11], is instead used in [2].

\section{Results and discussion}

\subsection{Steady-state evaporation}

The model proposed in the previous section, which relaxes the assumption of constant gas density and diffusion coefficients, has been used to calculate the evaporation rate for different liquid species evaporating in gaseous environment, covering a wide range of operating conditions (fire control, internal combustion engines, etc.). A parametrical analysis has been performed under steady state conditions and the results from the present model are compared with the classical model [19], found by solving the above reported species conservation equations under the hypotheses that $\rho$ and $D_{10}$ are independent of temperature, using the '1/3-rd rule' [11] to calculate the reference temperature 
and species mass fractions. To better appreciate the comparison among the different models, the evaporation rate is reported in non-dimensional form:

$$
\Psi_{*}=\frac{Y_{*}}{Y_{7 / 4}}
$$

where the variable property model with $n=7 / 4$ is taken as reference case. The non-dimensional vaporisation rate predicted by the classical model and by the present one, with $n=3 / 2$, are compared with the results from the present model, with $n=7 / 4$.

The comparison has been made selecting six evaporating fluids (water, acetone, ethanol, n-hexane, n-octane and n-dodecane). All the results presented in the following paragraphs are obtained under steady-conditions at 1 bar, setting $y_{v, \infty}=0$.

Figure 1 shows the effect of liquid temperature, which has been varied from $280 \mathrm{~K}$ up to a value close to the boiling temperature of each liquid species, on the non-dimensional evaporation rate parameter $\Psi$ (equation 37 ) for the selected six species evaporating in hot air at 500K. The effect of convection is also investigated varying the Reynolds number from 0 up to 100. The results show that the classical model, imposing constant density and diffusion coefficients, predicts different evaporation rate compared to the proposed model, with $n=7 / 4$, while the the proposed model with $n=3 / 2$ does not show remarkably differences for the whole range of operating conditions. Higher differences, up to about 10\%, are reported for acetone, ethanol and n-dodecane drops particularly at higher drop temperature, close to boiling conditions, while lower differences appear with water drops (up to about $2 \%$ ). The effect of convection is not that important and it slightly reduces the differences among the models, particularly at higher drop temperature.

This trend is confirmed increasing the gas temperature at $1500 \mathrm{~K}$ and the results are reported in figure 2 for all the six species, drop temperature and Reynolds number conditions of figure 1. The increase of the gas temperature enhances the differences among the models, for all the selected test cases, with the classical model predicting higher evaporation rate, up to about $30 \%$ when compared to the present one, with acetone drops evaporating in stagnant air $(\operatorname{Re}=0)$. The proposed model with exponent $n=3 / 2$ slightly overestimates the evaporation rate compared to the reference case, with a maximum difference up to about $12 \%$ with acetone and n-hexane drops close to boiling conditions. Again the effect of Reynolds number is not substantial, particularly when the two variable property models, proposed here, are compared. 
This motivates the choice to use the variable property model with exponent $n=7 / 4$ for the model assessment against experimental data bases available in the scientific literature, which is discussed in the following section.

\subsection{Comparison with experimental data}

This paragraph reports the comparison of the predictions obtained by the proposed variable property model with exponent $n=7 / 4$, described in the previous section, with the experimental data of liquid drops evaporating in air under transient conditions. Table 1 reports the operating conditions for all the test cases used for the model assessment. For all the investigated test cases the liquid drop initially experiences an heating-up process, where the liquid temperature increases and the evaporation is rather moderate at the beginning, then, once the drop temperature has reached an almost asymptotic value, which also depends on the gas temperature, the evaporation rate reaches higher values.

Figure 3 shows the transient evaporation of a decane drop evaporating in air at atmospheric pressure with temperature equal to $1000 \mathrm{~K}$, under convective conditions, with the Reynolds number initially equal to 17 . The graph reports both the transient non-dimensional radius and the drop temperature as predicted by the proposed model and as measured by [27]. A rather good agreement is confirmed between the model predictions and the experimental data, which were measured with a tolerance of $2.5 \%$ for the drop size, while the resolution of the temperature acquisition system was $1 \mathrm{~K}$ [27].The model is capable to reproduce the initial heat-up period, when the drop evaporation is rather low, and the successive evaporating period. The slop of the drop size reduction as predicted by the model is in very good agreement with the experimental measurements. Please notice that the reported experimental values of liquid temperature refers to the drop center, with differences of few degrees within the drop as reported in [27], while the proposed model assumes an infinite liquid thermal conductivity, neglecting any internal temperature gradients.

The capability of the model to reproduce the evaporation under different gaseous temperatures is shown in figure 4, which reports the transient profiles of the drop diameter square for hexane drops vaporizing under convective conditions, with the relative velocity fixed equal to $1 \mathrm{~m} / \mathrm{s}$, following the experimental data base of [28], where the accuracy of drop size measurements was equivalent to $0.01 \mathrm{~mm}$. Again the proposed model is capable to reproduce the drop size reduction, showing a rather good agreement with the experimental data for all the three test cases 
selected.

Finally figure 5 reports the non-dimensional drop diameter square, as function of time (divided by the initial drop diameter square) for heptane drop vaporising under different gaseous temperatures, from $471 \mathrm{~K}$ up to $741 \mathrm{~K}$. The experimental data base of [29] is used for model assessment. As reported in [29] the drop evaporation was assumed under almost quiescent atmosphere, although an accurate measurement of the relative velocity was not available, suggesting that the assumption of Reynolds number equal to 0 may be questionable. This motivates the choice to impose different initial Reynolds numbers in the model calculations (from 0 up to 4), to check its effect on the evaporation rate. The results reported in figure 5 confirm the not-negligeable effect of small convection on the drop size transient profile, suggesting that with the choice of the initial Reynolds number equal to 4 the model prediction well agree with the experimental data.

Table 1. Operating conditions for model comparison with experimental data bases.

$\begin{array}{lllll}\text { References } & \text { Species } & D_{d, 0}(\mathrm{~mm}) & T_{\infty}(\mathrm{K}) & R e_{0} \\ \text { Wong and Lin, 1992 [27] } & \text { Decane } & 1.961 & 1000 & 17 \\ \text { Downing, 1966 [28] } & \text { Hexane } & 1.648 & 437-546 & 48-66 \\ \text { Nomura, 1966 [29] } & \text { Heptane } & 0.7 & 471-741 & \sim 0\end{array}$

\section{Conclusions}

Being the exact calculation of the thermophysical properties a crucial aspect in modelling of drop evaporation for spray investigation, an analytical model has been developed predicting the heating and evaporation rates from a single component spherical drop solving the species, energy and momentum conservation equations in a radial coordinate system, explicitly accounting for the temperature dependence of mixture density and diffusion coefficient. The predictions from the model are compared with those obtained by the classical approach, which imposes constant density and diffusion coefficient, under steady state conditions for different evaporating species, drop and gas temperatures. The results enlighten that the differences among the different approaches are noticeable for acetone, ethanol and heavier hydrocarbons particularly at high gas temperatures. The effect of convection is also included in the model proposing a modified correlation of the Nusselt number, which is valid under low and high evaporating and convective conditions. 
The proposed model is validated against various data bases from the existing scientific literature, describing the transient size and temperature evolution of liquid drops at moderately high evaporating conditions. The good agreement between the model predictions and the experimental measurements confirms the validity of the proposed model for the selected range of operating conditions.

\section{Appendix}

The integral to be calculated is:

$$
I_{n}=\int_{\eta}^{1} \tilde{T}^{1-n}(z) d z
$$

where $\eta=\eta_{3}=\frac{\zeta_{3}-\zeta_{2}}{1-\zeta_{2}}$ for the convective case, while $\eta=0$ for the case with $\mathrm{Re}=0$. When $K_{\zeta_{2}} \neq 0$ a change of variable: $x=\left(\frac{\tilde{T}}{\left|K_{\zeta_{2}}\right|}\right)^{2-n}$ yields the following form:

$$
I_{n}=-\frac{1}{\check{Y}}\left|K_{\zeta_{2}}\right|^{1-n} \frac{1}{(2-n)} \begin{cases}\int_{x(\eta)}^{x_{s}} \frac{d x}{\left[x^{1 /(2-n)}-1\right]} & \text { for } K_{\zeta_{2}}>0 \\ \int_{x(\eta)}^{x_{s}} \frac{d x}{\left[x^{1 /(2-n)}+1\right]} & \text { for } K_{\zeta_{2}}<0\end{cases}
$$

while when $K_{\zeta_{2}}=0$ the integral reduces to:

$$
I_{n}=-\frac{1}{\grave{Y}} \int_{\tilde{T}(\eta)}^{\tilde{T}_{s}} \tilde{T}^{-n} d \tilde{T}
$$

The analytical solution is then, for $K_{\zeta_{2}} \neq 0$ :

$$
I_{n}\left(\eta, K_{\zeta_{2}} \check{Y}\right)=-\frac{1}{\check{Y}} \frac{\left|K_{\zeta_{2}}\right|^{1-n}}{(2-n)}\left\{W\left(\left(\frac{\tilde{T}_{s}}{\left|K_{\zeta_{2}}\right|}\right)^{2-n}\right)-W\left(\left(\frac{\tilde{T}(\eta)}{\left|K_{\zeta_{2}}\right|}\right)^{2-n}\right)\right\}
$$

where $W(x)$ is given by:

$$
W_{n}(x)=\left\{\begin{array}{ccc}
-\frac{1}{4}\left[2 \arctan (x)+\log \frac{x+1}{x-1}\right] & K_{\zeta_{2}}>0 & \text { for } n=7 / 4 \\
\frac{1}{4 \sqrt{2}}\left[2 \arctan (1+\sqrt{2} x)-2 \arctan (1-\sqrt{2} x)+\log \frac{x^{2}+\sqrt{2} x+1}{x^{2}-\sqrt{2} x+1}\right] & K_{\zeta_{2}}<0 & \\
-\frac{1}{2} \ln \left(\frac{1+x}{1-x}\right) & K_{\zeta_{2}}>0 & \text { for } n=3 / 2 \\
\arctan (x) & K_{\zeta_{2}}<0 & \\
2\left[x^{1 / 2}+\ln \left(x^{1 / 2}-1\right)\right] & K_{\zeta_{2}}>0 & \text { for } n=0 \\
2\left[x^{1 / 2}-\ln \left(x^{1 / 2}+1\right)\right] & K_{\zeta_{2}}<0 &
\end{array}\right.
$$

and for $K_{\zeta_{2}}=0$ :

$$
I_{n}=-\frac{1}{\check{Y}} \int_{\tilde{T}(\eta)}^{\tilde{T}_{s}} \tilde{T}^{-n} d \tilde{T}=-\frac{1}{\bar{Y}(1-n)}\left[\tilde{T}_{s}^{(1-n)}-\tilde{T}^{(1-n)}(\eta)\right]
$$


for $n \neq 1$. To notice that for the case with $R e=0$, the same equations can be used setting $\eta=0$ and $K_{\zeta_{2}}=K_{0}$.

\section{References}

[1] Sazhin, S.S., Droplet and sprays, Springer, 2014.

[2] Abramzon, B., Sirignano, W.A., Droplet vaporization model for spray combustion calculations, International Journal of Heat and Mass Transfer, 32 (9) (1989) 1605-1618.

[3] Tonini, S., Cossali, G.E., A novel vaporisation model for a single-component drop in high temperature air streams, International Journal of Thermal Sciences, 75 (2014) 194-203.

[4] Tonini, S., Cossali, G.E., One-dimensional analytical approach to modelling evaporation and heating of deformed drops, International Journal of Heat and Mass Transfer, 97 (2016) 301-307.

[5] Sazhin, S.S., Krutitskii, P.A., Gusev, I.G., Heikal, M.R., Transient heating of an evaporating droplet, International Journal of Heat and Mass Transfer, 53(13) (2010) 2826-2836.

[6] Sazhin, S.S., Krutitskii, P.A., Gusev, I.G. and Heikal, M.R., Transient heating of an evaporating droplet with presumed time evolution of its radius,International Journal of Heat and Mass Transfer, 54(5) (2011) 1278-1288.

[7] Sirignano, W.A., Fluid Dynamics and Transport of Droplets and Sprays, 2nd ed., Cambridge University Press, 2010 .

[8] Castanet, G., Lebouché, M., Lemoine, F., Heat and mass transfer of combusting monodisperse droplets in a linear stream, International Journal of Heat and Mass Transfer, 48 (2005) 3261-3275.

[9] Maqua, C., Castanet, G., Grisch, F., Lemoine ,F., Kristyadi, T., Sazhin, S.S., Monodisperse droplet heating and evaporation: experimental study and modelling, International Journal of Heat and Mass Transfer, 51(15-16) (2008) 3932-3945.

[10] Gusev, I.G., Krutitskii, P.A., Sazhin, S.S., Elwardany, A.E., New solutions to the species diffusion equation inside droplets in the presence of the moving boundary, International Journal of Heat and Mass Transfer, 55(7) (2012) 2014-2021. 
[11] Yuen, M.C., Chen, L.W., On Drag of Evaporating Droplets, Combustion Science Technology, 14 (1976) 147-154.

[12] Wannier, G.H., Statistical Physics, J.W.\& Sons, 1966.

[13] Fuller, E.N., Schettler, P.D., Giddings, J.C., New method for prediction of binary gas-phase diffusion coefficients, Industrial and Engineering Chemistry, 58(5) (1966) 18-27.

[14] Schlottke, J., Weigand, B., Direct numerical simulation of evaporating droplets, Journal of Computational Physics, 227 (2008) 5215-5237.

[15] Banerjee, R., Numerical investigation of evaporation of a single ethanol/isooctane droplet, Fuel, 107 (2013) 724-739

[16] Ghata, N., Shaw, B.D., Computational modeling of the effects of support fibers on evaporation of fiber-supported droplets in reduced gravity, Int J Heat Mass Transfer, 77 (2014) 22-36.

[17] Strotos, G., Malgarinos, I., Nikolopoulos, N., Gavaises, M., Predicting the evaporation rate of stationary droplets with the VOF methodology for a wide range of ambient temperature conditions, International Journal of Thermal Sciences, 109 (2016) 253-262.

[18] J.C. Slattery, Momentum, energy and mass transfer in continua, second ed., Edition R. Krieger Publ., New York, 482, 1981.

[19] N.A. Fuchs, Vaporisation and droplet growth in gaseous media, Pergamon Press, London, 1959.

[20] Tonini, S., Cossali, G.E., A multi-component drop evaporation model based on analytical solution of StefanMaxwell equations, International Journal of Heat and Mass Transfer, 92 (2016) 184-189.

[21] Tonini, S., Cossali, G.E., An analytical model of liquid drop evaporation in gaseous environment, International Journal of Thermal Sciences, 57 (2012) 45-53.

[22] Bird, R.B., Stewart, W.E., Lightfoot, E.N., Transport Phenomena, John Wiley \& Sons, New York, 1960.

[23] Frössling, N., The evaporation of falling drops (in German), Gerlands Beiträge zur, Geophysik, 52 (1938)170-216. 
[24] M.C. Yuen, L.W. Chen, Heat transfer measurements of evaporating liquid droplets, Innternational Journal of Heat and Mass Transfer, 21 (1978) 537-542.

[25] Renksizbulut, M., Yuen, M.C., Experimental study of droplet evaporation in a high temperature air stream, Journal of Heat Transfer, 105 (1983) 384-388.

[26] Dwyer, H.A., Calculations of droplet dynamics in high temperature environments, Progress in Energy and Combustion Science, 15(2) (1989) 131-158.

[27] Wong, S.C., Lin, A.C., Internal temperature distributions of droplets vaporizing in high-temperature convective flows, Journal of Fluid Mechanics, 237 (1992) 671-687.

[28] Downing, C.G., The evaporation of drops of pure liquids at elevated temperatures: rates of evaporation and wet-bulb temperatures, AIChE Journal, 12 (4) (1966) 760-766.

[29] Nomura, H., Ujiie, Y., Rath, H.J., Sato, J.I., Kono, M., Experimental study on high pressure droplet evaporation using microgravity conditions, Symposium (international) on combustion (1996) 1267-1273. 


\section{$8 \quad$ Figure captions}
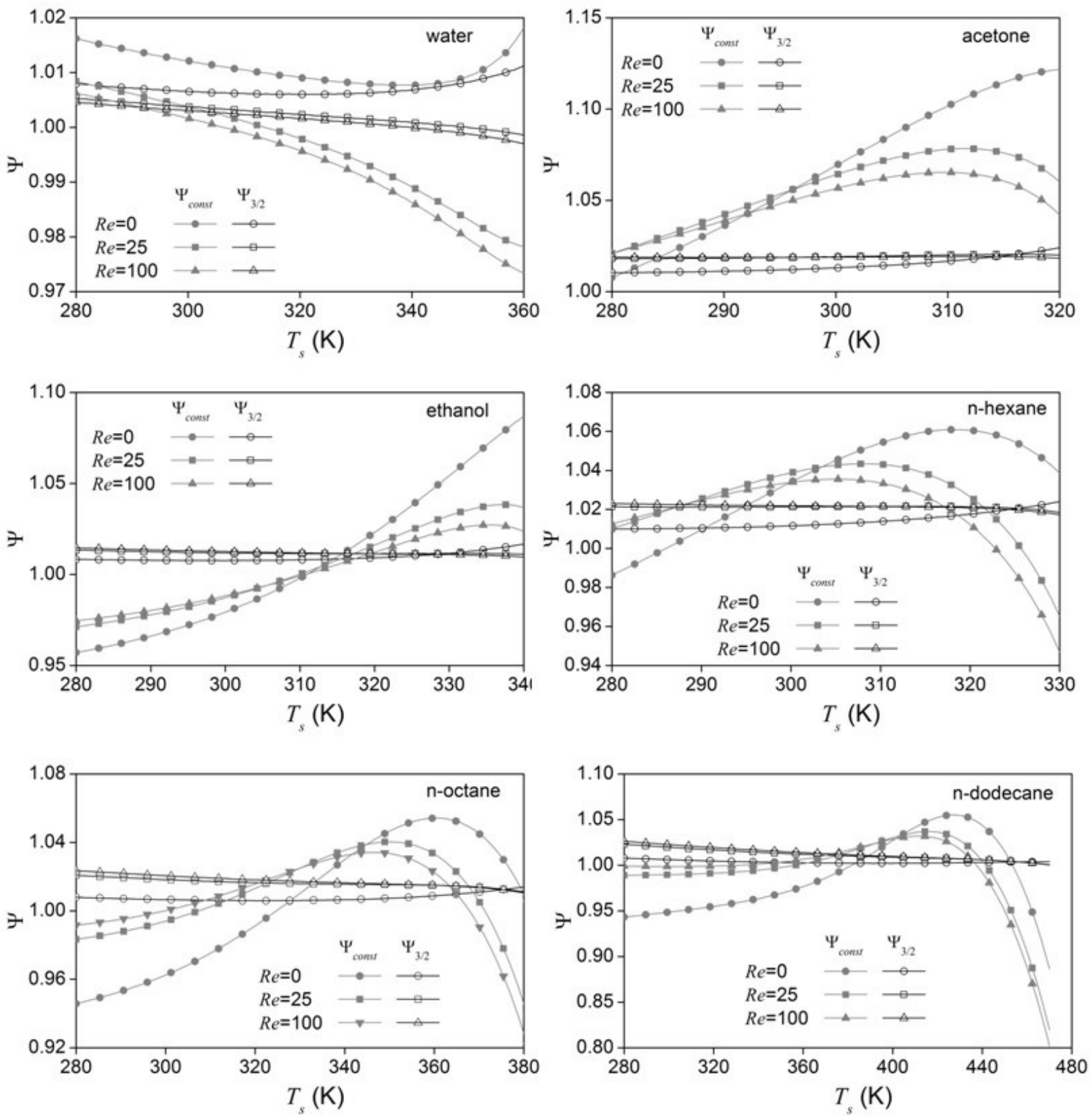

Figure 1. Effect of liquid temperature and convective conditions on the drop evaporation rate ratio calculated by the classical model and by the present model with $n=3 / 2$, compared with the present model with $n=7 / 4$, for six liquid species evaporating in air at 500K. 

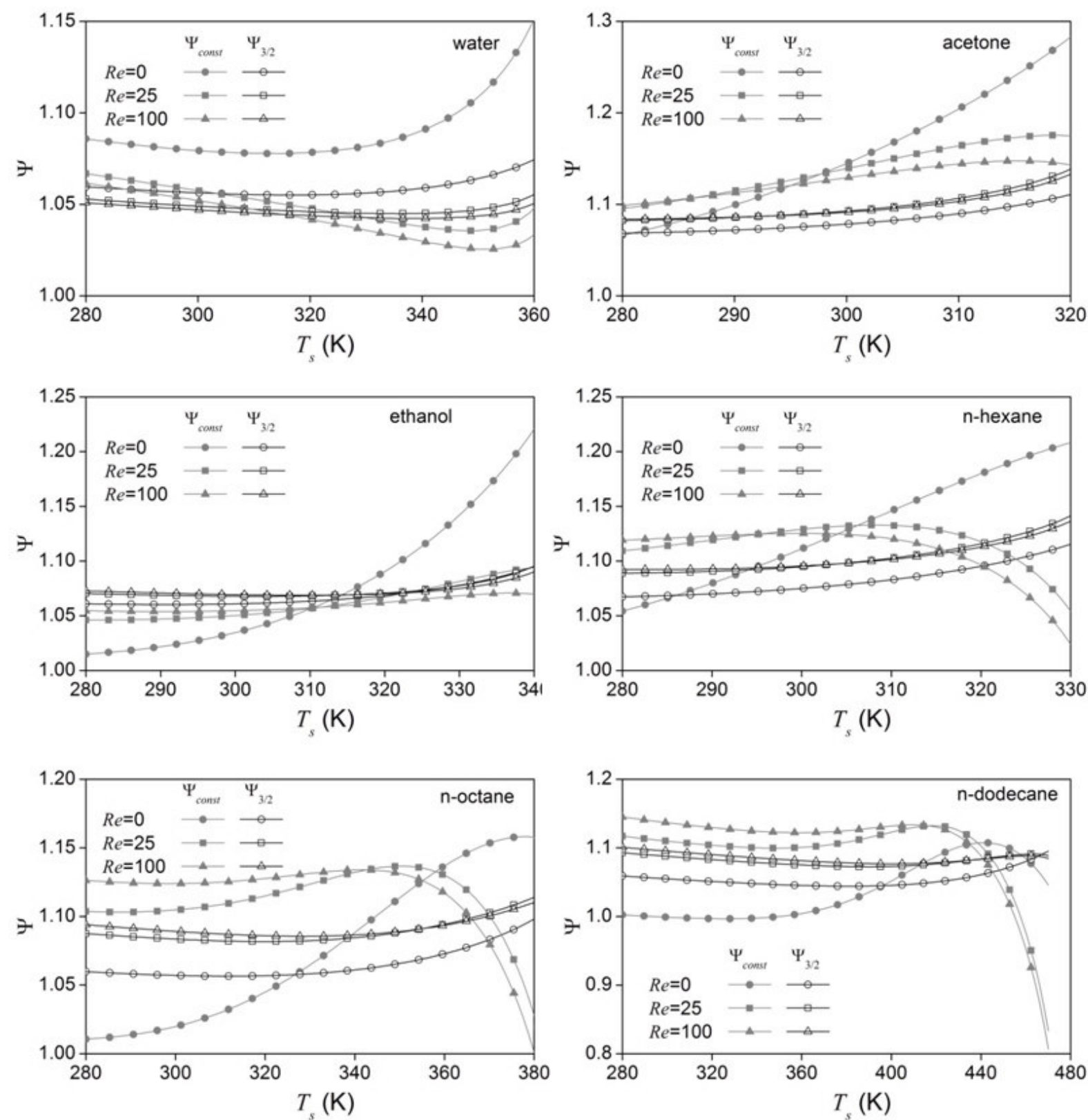

Figure 2. Effect of liquid temperature and convective conditions on the drop evaporation rate ratio calculated by the classical model and by the present model with $n=3 / 2$, compared with the present model with $n=7 / 4$, for six liquid species evaporating in air at 1500K. 


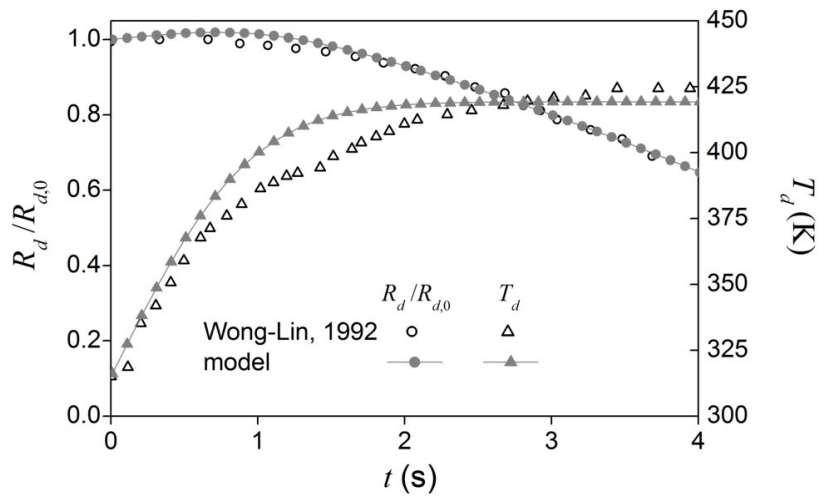

Figure 3. Comparison between the transient non-dimensional radius and drop temperature as predicted by the present model $(n=7 / 4)$ and from the experimental measurements of Wong and Lin [27] for n-decane drop evaporating in air at $1000 \mathrm{~K}, 1$ bar and $\operatorname{Re} 0=17$.

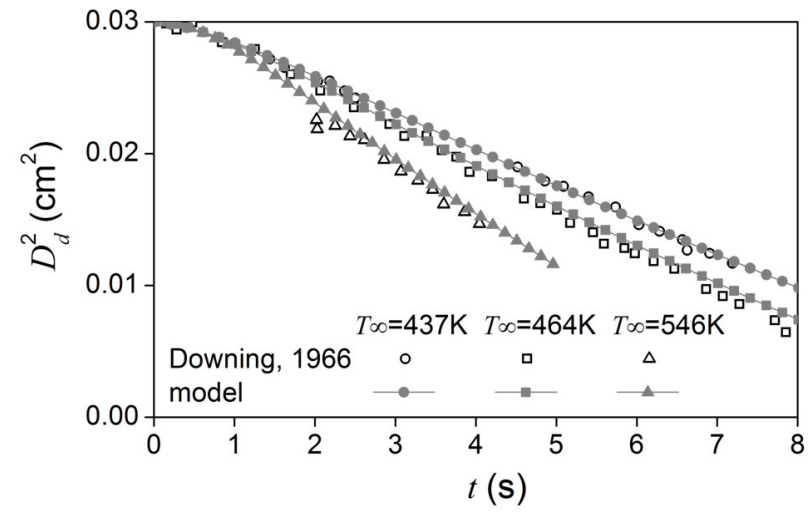

Figure 4. Comparison between the transient profiles of the drop diameter square as predicted by the present model ( $n=7 / 4$ ) from the experimental measurements of Downing [28] for n-hexane drop evaporating in air at 1 bar pressure, temperature equal to $437 \mathrm{~K}, 464 \mathrm{~K}$ and $546 \mathrm{~K}$, and velocity equal to $1 \mathrm{~m} / \mathrm{s}$. 

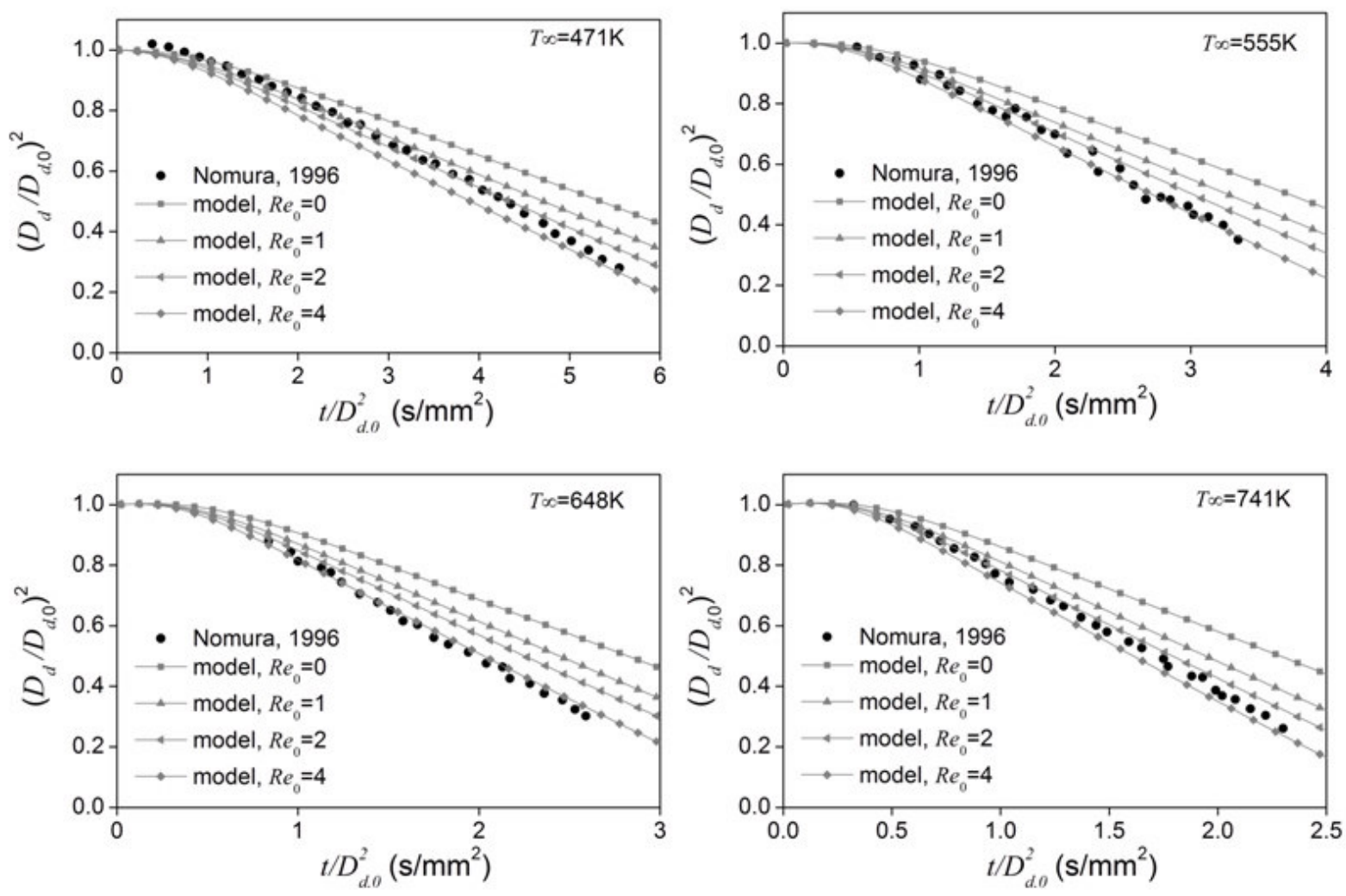

Figure 5. Comparison between the transient profiles of the non-dimensional drop diameter square as predicted by the present model $(n=7 / 4)$ from the experimental measurements of Nomura [29] for n-heptane drop evaporating in air at 1 bar and temperature equal to $471 \mathrm{~K}, 555 \mathrm{~K}, 648 \mathrm{~K}$ and $741 \mathrm{~K}$. In the absence of the exact measurement the initial Reynolds number, its value has been varied from 0 to 4 in the calculations. 